\title{
Utilizing Six Sigma Methodology for Training Undergraduate Student for Conducting Global Field Research
}

\author{
Kritika Chopra \\ Email: kchopra@iastate.edu \\ Shweta Chopra, $\mathrm{PhD}$ \\ Email: schopra@iastate.edu \\ Chad Laux, PhD \\ Email: claux@purdue.edu
}

Department of Agricultural and Biosystems Engineering, Iowa State University

Department of Technology Leadership and Innovation, Purdue University

\begin{abstract}
Purpose: With the increase in demand for the global research, scholars in engineering and technology discipline do not hesitate in taking up global opportunity for conducting research. Training the next generation for such international research opportunity is key and involving undergraduate students' beyond study abroad is important.
\end{abstract}

Design/Methodologies/Approach: This research focuses on improving the efficiency of agricultural supply chain by addressing the challenge of post-harvest losses faced by various stakeholders in Public Distribution System of Chhattisgarh, India.

Findings: We identified the potential use of Six Sigma methodology along with quality management tools for successful implementation of a research project in undergraduate curriculum and address barriers in field research.

Practical Implications: This research highlights the potentials and challenges of conducting research in global setting. Additionally, the authors have utilized quality management tool and developed a model that can be adapted by engineering and technology scholars to conduct research effectively in global environment.

Originality: This study was done though an undergraduate research model with close faculty mentorship.

Keywords: undergraduate student, quality management tools, Six Sigma, India, global research, DMAIC

Paper Type: Research 


\section{Introduction}

Research can be defined as systematic investigation of a topic which explores the prior work conducted by scholars and can be systematically disseminated. Furthermore, research is based on facts, data collection and analysis, along with a systematic review of literature. Data collection can be done from variety of resources, in both qualitative and quantitative methods using formal/informal or other innovative techniques. Research should have an impact on to a group of scholars or general public, provided data based interpretation of scholarly work. Most often research will also address a gap in the existing literature and advanced an existing theory by providing significant proof for any claims made.

Training next generation for the future endeavor is one of the responsibility academician has. Undergraduate students have many fresh ideas which often time researchers who are embedded in the field are unable to see. Often time gaining those idea can turn out very fruitful for the research and training purposes. Utilizing six-sigma framework will provide a basis for establishing SOP's for training future students to become successful scholars.

\subsection{Research Gap and Research Question}

Six sigma methodology has been successfully employed in the manufacturing industry and tremendous benefit has been gained through it. It is still very novel to utilize Six Sigma evaluation for service industry and there is research scarcity in the field of use of Six Sigma in education. We believe that Six Sigma methodology has a huge potential to be employed in education and our undergraduate students can get significantly benefited from this systematic training. Furthermore, it can help create future researcher and provide a path for continuous improvement practice in field research.

\section{Six Sigma and Educational Research}

There are various methodologies available for constructing a relevant framework for implementing systematic change in an organization. Six Sigma's DMAIC (define - measure analyze - improve - control) methodology is an important approach for improving, augmenting $\&$ aligning a process with respective business objectives. Where the Define phase is used to delineate a problem, objectives, project scope, requirements or expected outcomes of the project based upon the customer voice, which in this case was the successful conduct of undergraduate project in the field. Measure phase helped to establish a baseline and collect data on the existing situation which helped us recognize the potential challenges and various reasons for them in field work utilizing undergraduate research participation. Analyze phase helps us to evaluate these significant reasons and concentrate on the most important ones. The analyze phase also helps us in finding the potential correlations and the extent of the dependency between the cause and the effect. The improve phase helps us to identify the processes which can help us overcome constraints, using various techniques of brainstorming used to identify potential solutions. The control phase may be used to sustain these improvements achieved in the previous phase, which again be a part of continuous improvement.

This model fits with our current challenge of training undergraduate students to effectively conduct field work and meaningfully contribute to the research and development projects by identifying the prominent challenges and brainstorming to find implementable solutions; with particular attention to gauging improvements in implementation(Zahra and George, 2002). An 
integration of the DMAIC with Kaizen (a practice of continuous improvements) can yield effective results for a sustainable impact.

Six Sigma DMAIC methodologies have been used in the past for the education delivery in the higher education to enhance students learning outcome (Antony et al., 2012). Six Sigma tools have also been suggested for increasing the learning outcomes, retention and recruitment of students by using them to address the behavior of the process by driving the continuous improvement. Researchers have also successfully demonstrated the application of DMAIC for continuous improvement and training by customizing curriculum (Antony and Banuelas, 2002).

\section{Utilizing Quality Control Tool for Systematically Analyzing Challenges}

Every problem or challenge faced can be derived into the multiple factors causing it. Thus, the problem or challenge may be resolved by tackling the individual factors separately (Doggett, 2005). If the causes are not identified in solving a problem, then the solution would only address the symptoms instead of the problem itself (Wahed et al., 2010). Root-Cause Analysis (RCA) is the method in which, the problematic process is identified and it is broken-down into factors causing the problem, when these factors are resolved, the errors in the process are resolved and will not recur (Doggett, 2005). These tools help users to identify the factors of the cause, through a structured approach, accurately and efficiently. Figure 1 describes systematic analysis of Sixsigma methodology for undergraduate training.

\subsection{Root-Cause Analysis Tools}

Three generic tools may be used to identify root causes. The tools are cause-and-effect diagram (CED) also known as fishbone diagram, the interrelationship diagram (ID), and the current reality tree (CRT). These tools are frequently used to identify the problems, their solutions and make decisions about solving the causes by evaluating the benefits and the cost-effectiveness when the solution is implemented.

Fishbone diagrams or CEDs are used to demonstrate the causes of a certain problem through a pictorial representation. The causes are sorted and related to the problem using a classification schema that are specified in the steps of creation of a CED (Doggett, 2005). The dispersion CED, the process classification CED, and the cause enumeration CED are three types of CEDs. These CEDs can be differentiated by their process of selecting the causes for the problem and their way of visually representing these causes.

\subsection{Prioritization}

Every individual, every field and even every process has an established list of priorities. Prioritization is a critical requirement in any system. However, establishing prioritization in a system may be a challenging task. This is because the reasons behind prioritizing are different for different scenarios (Lehtola et al., 2004). A requirement of prioritization can be generally of two types, the first one is prioritization due to the implementation order and the second one is prioritization due to importance (Firesmith, 2004). Both implementation order and importance priority paths may relate and have three different categories of priority to establish assessment. High, Medium and Low scales are one of the sets of the prioritization scale. In this study prioritization is done by importance and the scales that are used in prioritization are high, medium and low. 
Above described quality management tools will help address the challenges associated with designing effective undergraduate research methods and implementing them in the field. These quality management tools along with Six Sigma methodology will help systematically address the research methods obstacles and sustain student research work.

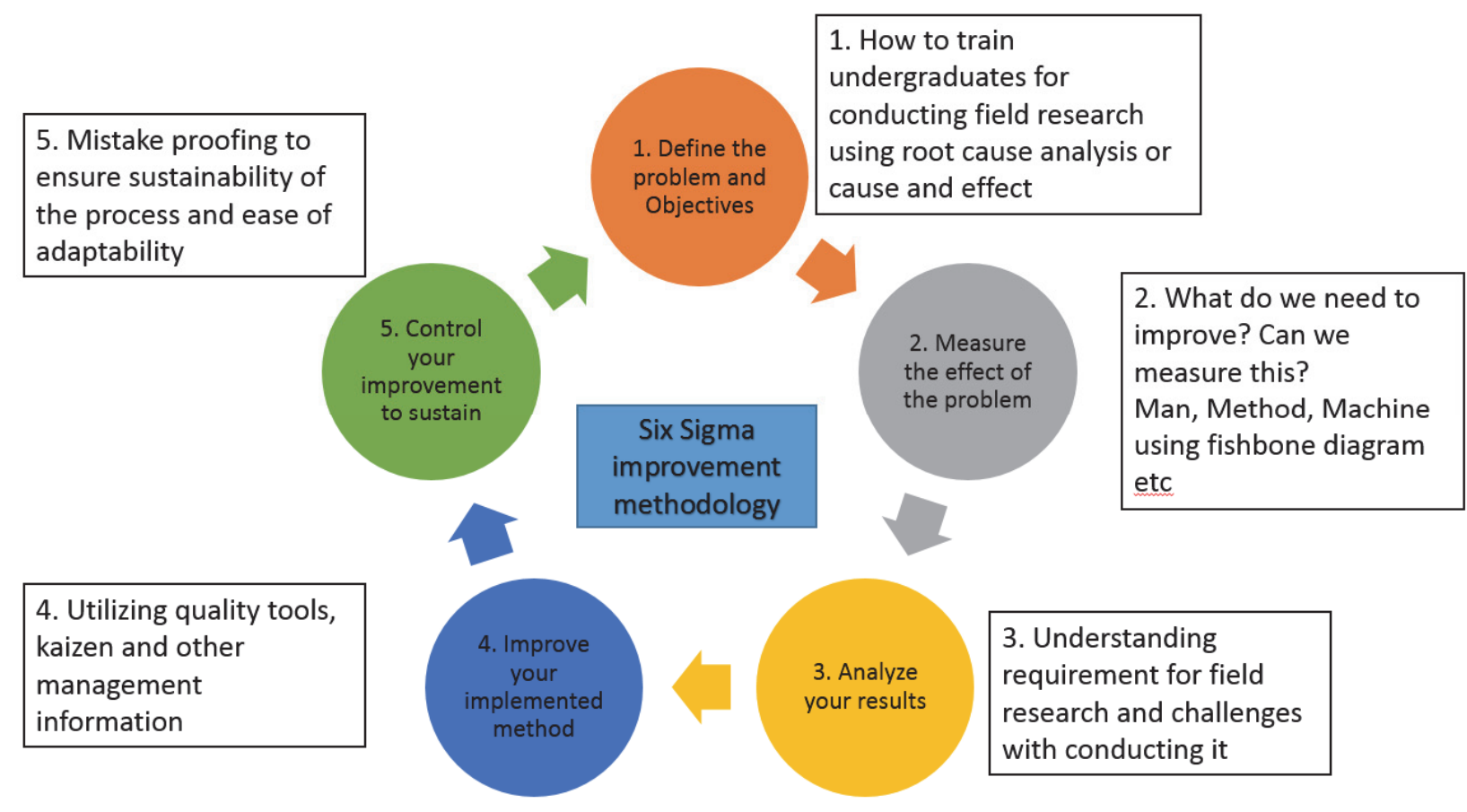

Figure 1. Six Sigma Process Improvement Cycle for Training Undergraduates

\section{Conclusion}

Six Sigma (DMAIC) approach helped the authors to systematically design a research project flow (Seow and Antony, 2004). DMAIC exhibits potential for future use in undergraduate research studies in education. With the guidance of professors as mentors, the professor models to the undergraduate student the skills to be a leader in problem solving, oriented toward research. There is a positive relationship between undergraduate students conducting research to further their higher education to graduate school. University support of undergraduate research assistantships (URA) are available to a majority of students. See Table 1 which describes utilization of Six Sigma Methodology for building research by training undergraduate student and collecting field research. This allows the student to try real-world practices and techniques without great consequence and contribute to the academy. 


\begin{tabular}{|l|l|l|}
\hline $\begin{array}{c}\text { Six Sigma } \\
\text { Methodology }\end{array}$ & \multicolumn{1}{|c|}{ By research group } & \multicolumn{1}{|c|}{ In the field } \\
\hline Define & $\begin{array}{l}\text { Undergraduate was debriefed about } \\
\text { the project which will be conducted } \\
\text { in the field }\end{array}$ & $\begin{array}{l}\text { Field sight was chosen for PICS project } \\
\text { initial understanding }\end{array}$ \\
\hline Measure & $\begin{array}{l}\text { Student success will depend on } \\
\text { number of participant interviewed } \\
\text { or survey filled }\end{array}$ & $\begin{array}{l}\text { Survey was designed to measure the } \\
\text { current perspectives of the field }\end{array}$ \\
\hline Analyze & $\begin{array}{l}\text { Challenges faced by the student in } \\
\text { the field was analyzed by } \\
\text { conference call with the student and } \\
\text { faculty mentor }\end{array}$ & $\begin{array}{l}\text { Survey was filled out by participant } \\
\text { and result was analyzed for PICS } \\
\text { project }\end{array}$ \\
\hline Improve & $\begin{array}{l}\text { Proper guidance was given to } \\
\text { student to address the challenges } \\
\text { faced }\end{array}$ & $\begin{array}{l}\text { Based upon the analysis of results } \\
\text { researcher understood PICS bags will } \\
\text { be useful to be used in the field }\end{array}$ \\
\hline Control & $\begin{array}{l}\text { Plan for winter field trip with } \\
\text { undergraduate student and mentor } \\
\text { was developed and successfully } \\
\text { completed }\end{array}$ & $\begin{array}{l}\text { First experiment with PICS bag rice } \\
\text { storage was conducted in December } \\
\text { 2017 }\end{array}$ \\
\hline
\end{tabular}

Table 1. Describes the Use of Six Sigma Methodology for Building Research by Training Undergraduate Student and Collecting Field Research

By utilizing Six Sigma as an undergraduate research approach, benefits can be obtained in conducting field work and training undergraduate students. Conducting field research is a rewarding and challenging endeavor (May, 2011). In today's global economy, it is becoming less common for one in the academy to not work with, or teach, someone from another nation and/or culture. As students are being educated to learn about the global community, academics are discovering the same advantages that results in new combinations, connections, and networks of ideas that result in novel approaches, especially in the technical and engineering areas. DMAIC could be adapted to mitigate the barriers of interdisciplinary work by focusing on the analytic and creative aspects of Six Sigma. Regardless of the perspective, the endeavor serves meaning to the individual.

One of the implications of this research is that major portion of it is based of perception of one undergraduate student and two faculty. This research is focused on agriculture research in India and some of the findings might not hold true for the research to be conducted in the other part of the world. It is important to keep in mind that this research was conducted by an undergraduate student in US University who is an Indian national and knows the local language very well. This research experience and gaining field access will vary tremendously for a researcher who is not from the nation or doesn't know the local culture or language. For future work it will be important to gain input from multiple student and specifically students who have either been to multiple research site. That will help develop best practices applicable in different fields. It will be very interesting to gain perspective of a student who does not belong to the country where they are going to conduct field work. Compiling their challenges and addressing ways to overcome those will be a great contribution to the research field. 


\section{References}

Antony, J., Banuelas, R., 2002. Key ingredients for the effective implementation of Six Sigma program. Measuring business excellence 6, 20-27.

Antony, J., Krishan, N., Cullen, D., Kumar, M., 2012. Lean Six Sigma for higher education institutions (HEIs) Challenges, barriers, success factors, tools/techniques. International Journal of Productivity and Performance Management 61, 940-948.

Doggett, A.M., 2005. Root cause analysis: a framework for tool selection. The Quality Management Journal 12, 34.

Firesmith, D., 2004. Prioritizing Requirements. Journal of Object Technology 3, 35-48. Lehtola, L., Kauppinen, M., Kujala, S., 2004. Requirements prioritization challenges in practice. Presented at the International Conference on Product Focused Software Process Improvement, Springer, pp. 497-508.

May, T., 2011. Social research. McGraw-Hill Education (UK).

Seow, C., Antony, J., 2004. Some pros and cons of six sigma: an academic perspective. The TQM Magazine 16, 303-306.

Wahed, M.A., Montaser, M., Sami, S.A., 2010. Root cause analysis for medical equipment calibration laboratory nonconformities. Presented at the Biomedical Engineering Conference (CIBEC), 2010 5th Cairo International, IEEE, pp. 206-209.

Zahra, S.A., George, G., 2002. International entrepreneurship: The current status of the field and future research agenda. Strategic entrepreneurship: Creating a new mindset 255-288. 